\title{
Alea iacta est. Efectos de la Claridad vs. Ambigüedad en la Meta y los Planes de Acción sobre el Logro de Metas
}

\section{Alea iacta est. Effects of Clarity vs. Ambiguity in the Goal and Action Plans on Goal Achievement}

\author{
Jesús María de Miguel Calvo, Pablo González Pascual y Amalio Blanco Abarca \\ Universidad Autónoma de Madrid
}

\begin{abstract}
Resumen. Se ha postulado que tener las metas claras conlleva una consecución eficiente de metas. En esta investigación analizamos si formular y tener las metas claras es una condición necesaria para alcanzar los objetivos. Las metas claras dieron lugar a un buen rendimiento sólo si se disponía de competencias específicas para realizar la tarea propuesta (Estudio 1); en el caso contrario, fue más eficaz una formulación de meta ambigua (Estudio 2). La introducción de una condición de planificación en el Estudio 3 refrenda que las metas claras no son siempre más eficaces, dado que en la condición de no-planificación fueron más efectivas las metas ambiguas. En cambio, cuando los participantes pudieron planificar sus acciones, no se hallaron diferencias entre las metas claras y ambiguas. Se discuten las implicaciones teóricas y aplicadas de los resultados.

Palabras clave: meta clara, meta ambigua, formulación de metas, logro de metas, planes de acción, equipos de trabajo.
\end{abstract}

\begin{abstract}
It has been established that having clear goals is a required condition to achieve objectives. In the following research we will analyze if having clear goals is a needed condition to achieve goal. Clear goal instruction triggered better performance only if the individual posses adequate competencies to develop task (Study 1); otherwise goal instruction was more effective (Study 2). When variable planning was added (Study 3 ) it verifies that clear goal setting are not always more effective, since ambiguous goal instruction was more effective under the condition of not planning. Whenever the individuals were able to plan their actions, there were differences found between clear goal instruction and ambiguous goal instruction. Theoretical and applied implications of the present findings were discussed.

Key words: clear goals, ambiguous goals, goal setting, goal achievement, action plans, team work.
\end{abstract}

Conseguir lo que deseamos es una motivación inherente a personas, grupos y organizaciones. Una de las primeras operaciones que realizamos para convertir nuestras aspiraciones en realidad consiste en traducir los deseos en objetivos claramente formulados. De hecho, comúnmente las teorías de metas plantean que tener metas claras es una condición necesaria para alcanzar los objetivos. La misma idea constituye uno de los postulados del planteamiento estratégico. Por ejemplo, las investigaciones han mostrado que las metas formuladas en términos específicos conllevan un rendimiento mejor que las metas imprecisas (Locke, Shaw, Saari, y Latham, 1981). Los estudios que aquí se presentan analizan si formular y tener metas claras es siempre una condición necesaria para alcanzar con éxito dichas metas. Además, examinaremos si los efectos diferenciales en el logro de metas, derivados de la claridad en su formulación, estarán modulados por su interacción con factores asociados a

Correspondencia: Jesús María de Miguel Calvo, Departamento de Psicología Social y Metodología, Facultad de Psicología, Universidad Autónoma de Madrid. C/ Ivan P. Pavlov, 6, 28049 Madrid, España. E-mail: jesus.demiguel@uam.es los esfuerzos para conseguirlas; concretamente con el dominio de ciertas competencias o la posibilidad de planificar. En tercer lugar, nos interesa indagar el efecto del ajuste (concordancia o discrepancia) entre la formulación de la meta (clara o ambigua) y la oportunidad de logro (que propicia la competencia o la planificación) en la consecución de metas. El alcance de estos estudios podrá tener especial relevancia en la metodología que grupos y organizaciones establecen para mejorar el logro de sus metas. Mayormente, cuando los grupos afrontan la necesidad de superar una previsible divergencia entre el contenido de la meta y el potencial para conseguirla.

El logro de metas ha sido objeto de estudio para distintas disciplinas y en el presente contexto de investigación cobran especial relevancia en primer lugar, las vinculadas a la perspectiva psicológica y, en segundo lugar, las derivadas de diversas aproximaciones a la organización formal. Las primeras han abordado el problema considerando, fundamentalmente, procesos cognitivos, motivacionales y volitivos. Las segundas, atendiendo especialmente a procesos estratégicos, de toma de decisiones, de solución de problemas y de mejora de la calidad. 
La perspectiva psicológica ha heredado una gran influencia de los estudios sobre niveles de aspiración de Lewin, Dembo, Festinguer y Sears (1944). Los autores identificaron dos tipos de problemas que las personas deben resolver para dirigir sus conductas hacia una meta. Uno afronta la necesidad de formular las metas y ajustarlas en función de la expectativa de logro ("goal setting"). El otro se refiere a los esfuerzos que las personas deben realizar para lograrlas ("goal striving"). Es decir, la consecución con éxito de las metas implica dos tareas: formular las metas y perseguirlas (Gollwitzer, 1990; Oettingen y Gollwitzer, 2001). Mientras que las teorías sobre la formulación de las metas se han centrado en el contenido y la estructura de las metas, las teorías sobre auto-regulación han hecho lo propio con la consecución con éxito de las metas.

La formulación de metas ha sido objeto de interés desde finales del siglo XIX hasta los años 60 del siglo XX. No obstante, la formulación de metas presentaba un uso esporádico en estudios caracterizados por su orientación a la intervención y la carencia de un marco teórico (Latham y Locke, 2007). Sólo recientemente se ha formulado una teoría integradora ("goal setting theory") por parte de Locke y Latham (Latham y Locke, 2007; Locke y Latham 2002). Locke y Latham describen el núcleo fuerte de su teoría en función de la especificidad y dificultad de las metas. Explican cómo las metas afectan a los resultados mediante cuatro mecanismos: a) ejercen una función directiva al guiar tanto la atención como los esfuerzos hacia las actividades que son relevantes para dicha meta; b) cumplen una función de activación ("energizing") en cuanto que las metas más difíciles conllevan mayores esfuerzos para alcanzar dicha meta; c) conllevan una persistencia mayor o menor en función de la dificultad de la meta; y d) afectan indirectamente a la acción al conducir el conocimiento y las estrategias relevantes para la consecución de la meta. Además, identificaron una serie de moderadores que influyen en los efectos del núcleo fuerte (tales como la el compromiso con la meta, la información sobre el progreso en los resultados y la complejidad de la tarea).

Por lo tanto, el contenido de las metas sustenta el núcleo fuerte de la teoría y constituye uno de los pilares de la investigación en este campo. Las dimensiones de dificultad y especificidad de las metas han sido objeto de numerosas investigaciones (Locke et al., 1981). En lo que respecta a los efectos de la claridad de las metas sobre el rendimiento, éstos apenas fueron estudiados hasta el comienzo de los años 80, tal y como apuntan Locke y colaboradores (1981). En cambio, en años posteriores han sido varias las investigaciones que se han centrado en esta dimensión. Así, por ejemplo, Kjormo y Halvari (2002) encontraron una relación positiva entre la claridad de metas y la cohesión grupal que, a su vez, afectaba favorablemente al rendimiento de deportistas. Recientemente se ha investigado cómo afecta un proceso de adquisición de una empresa a sus empleados (Nemanich y Keller, 2007). En este contexto, los autores encontraron que la claridad de meta se relacionaba positivamente con el rendimiento.

Por su parte, los problemas asociados a los esfuerzos para conseguir la meta han dado lugar a modelos como el de fases de acción o Modelo del Rubicón (Heckhausen y Gollwitzer, 1987; Gollwitzer, 1990, 1996). El modelo describe el curso de las distintas fases de acción que suceden para alcanzar una meta, considerando tanto los problemas de formulación como los de consecución de la meta. El modelo distingue cuatro fases secuenciales desde que se libera el impulso o deseo para conseguir el objetivo hasta su logro, adecuación o descarte: 1) predecisional, 2) post-decisional o preaccional, 3) de acción y 4) post-accional. Las cuatro fases quedan conectadas mediante tres momentos que ocurren en la transición de una a otra: una decisión que transforma el deseo en una intención de meta, un plan que conduce al inicio de la acción y un resultado objetivo que necesita una valoración interpretativa.

En la primera fase, predecisional, las personas deliberan entre los distintos deseos que les gustaría lograr. Dado que no pueden conseguir todos sus deseos, necesitan elegir a cuál de ellos dedicaran esfuerzos para lograrlo. La elección se realiza mediante un criterio evaluativo que considera dos aspectos: viabilidad y deseabilidad. La viabilidad se interpreta en términos de expectativa de logro. La deseabilidad se interpreta en términos de expectativa de valor. La fase predecisional concluye cuando el deseo se transforma en intención, es decir la motivación hacia la meta se transforma en voluntad de realizar un esfuerzo para conseguirla. Esta transición se caracteriza por el compromiso que adquiere el individuo para alcanzar la meta. El compromiso con la meta desencadena un proceso secuencial e irreversible que no admite vuelta atrás. La metáfora del "paso del Rubicón"1 sirve para explicitar este postulado: la meta que se forma en la primera fase pasa inexorablemente por las fases de planificación, ejecución y evaluación hasta que es lograda o descartada (Beckmann y Gollwitzer, 1987). En resumen: las personas valoran y priorizan sus deseos, deciden qué deseo van a lograr y deciden lograrlo, transforman el deseo en una intención de meta y se comprometen con la meta dando paso a la fase postdecisional.

Las acciones de la segunda fase, postdecisional o preacccional, se encaminan a la planificación. Se planifica dónde, cómo y cuándo. En esta fase se procesa la información relevante para lograr la meta elegida y reducir la

\footnotetext{
${ }^{1}$ Gollwitzer, Heckhausen y Ratajczak (1989) explican el uso de la metáfora del Rubicón: Pasar el Rubicón significa dar un paso hacia una meta sin poder retroceder. La metáfora del paso del Rubicón se toma de un hecho histórico. Julio César, cruzó el río Rubicón consciente de que ello desencadenaría la guerra civil en Roma y conciente que cruzado el río no tendría oportunidad de dar marcha atrás. Tras cruzar el río, Julio César dio la orden de avanzar a su ejército arengándolos con la frase alea iacta est (la suerte está echada). Suetonio (1992) los doce Césares. Madrid: Gredos.
} 
disonancia (Beckmann y Gollwitzer, 1987). Mientras en la fase predecisional se procesa la información con imparcialidad, en la fase postdecisional procesar la información de manera imparcial deja de ser funcional (Heckhausen y Gollwitzer, 1986). El plan propuesto conduce al inicio de las acciones dirigidas a lograr la meta, es la transición a la tercera fase. En la fase accional se ejecutan las acciones orientadas al logro de la meta. En la última fase, postaccional, la persona evalúa el logro de la meta considerando si el resultado previsto se ha alcanzado o no, en los términos esperados. La evaluación entre lo esperado y lo conseguido conlleva un nuevo proceso de deliberación y evaluación, en términos de viabilidad y deseabilidad, que proporcionan al sujeto información para tomar nuevas decisiones.

Respecto a la perspectiva de la organización formal, las metas se interpretan como un elemento de la estrategia. Para Quinn (1993) la estrategia integra tres elementos: a) las metas u objetivos que establecen el qué se va a lograr y el cuánto se va a lograr pero no el cómo se van a lograr; b) Las políticas, entendidas como reglas o guías que acotan los límites en los que se ejecutarán las acciones, y c) los programas que especifican la secuencias de la secuencia de acción que conducirá al logro de la meta. A su vez, la estrategia para ser eficaz debe cumplir una serie de criterios, entre ellos, la necesidad de definir los objetivos de forma clara.

En este mismo contexto, han sido muchos los planteamientos metodológicos que orientan el trabajo de grupos y equipos sobre la base de una secuencia organizada de acciones orientadas al logro de metas. La finalidad de dichos modelos es la de instaurar procedimientos que, aplicados de forma sistemática y con el concurso de diversas herramientas, faciliten a los miembros de una organización lograr los objetivos que persiguen. Entre ellos, cabe destacar el ciclo PDCA (Deming, 1982; Shewhart, 1931) y la metodología RADAR (European Fundation for Quality in Management, 2009). El ciclo PDCA distingue cuatro fases en el proceso de logro de metas. En la fase 1, Plan (planificar), se planifica la actividad a desarrollar, identificando los problemas y formulando el objetivo, se buscan las causas del problema y se idean los medios para resolverlos. En la fase 2, Do (hacer), se realiza lo planificado en la fase anterior. En la fase 3, Check (comprobar), se controla o verifica la actividad o actuaciones, introduciendo los ajustes o correcciones necesarios durante su desarrollo. La fase 4, Act (actuar), es una fase de evaluación una vez finalizadas las fases anteriores. Su finalidad es determinar si se corrigen o introducen modificaciones que mejoren los resultados o, en su caso, si se estandariza el proceso toda vez que los resultados alcanzados sean favorables. De esta forma, se pueden prever o planificar los posibles nuevos objetivos, comenzando de nuevo el Ciclo.

La metodología RADAR (Results, Approach, Deployment, Assessment and Refinement) guarda una estrecha relación con el ciclo PDCA. Ahora bien, se diferencian fundamentalmente en sus puntos de clausura y de cierre. En la metodología RADAR los resultados (results) -definir los resultados u objetivos que desean lograrse- se conciben al inicio; cerrando el ciclo con la evaluación y revisión (assessment and refinement) -evaluar los resultados realmente alcanzados por la organización-. Entre ellos se determinan dos momentos, enfoque (approach) y despliegue (deployment). El enfoque abarca la planificación de las acciones y la integración de la planificación con los objetivos de la estrategia, mientras que el despliegue se refiere a la puesta en marcha del enfoque de manera sistemática.

En resumen, bien originadas en teorías psicológicas o bien originadas en planteamientos organizacionales, se ha determinado que el logro de metas requiere de una serie de tareas dirigidas a la formulación y planificación de las mismas. Las que se complementan con tareas de evaluación de resultados, corrección de acciones y reformulación de objetivos. La formulación de la meta se ha asociado al logro de la misma bajo la condición de claridad. Pero poco sabemos sobre cómo actúan las personas y los grupos cuando afrontan situaciones donde no se dan las condiciones óptimas para el logro de sus objetivos.

Los estudios que presentamos tienen como objetivo contrastar si una instrucción de meta clara es una condición necesaria para alcanzar las metas propuestas, es más, suponemos que su logro está modulado por la disponibilidad de un plan de acción correspondiente. Las metas claras están conceptualizadas en las presentes investigaciones como aquellas que especifican un estado final deseado que no necesita ser interpretado, sino que dan lugar de forma inmediata a una iniciación de la acción. Las metas ambiguas, por otro lado, son aquellas que requieren de una elaboración posterior y pueden dar lugar a diferentes interpretaciones. Planteamos que ciertas situaciones ambiguas o discrepantes pueden resultar exitosas dado que las personas y los grupos tienden a superar la inconsistencia y buscan un equilibrio cognitivo (p.e., Festinger, 1957; Abelson et al., 1968). En definitiva, proponemos contrastar las siguientes hipótesis:

Hipótesis 1: las metas claras conllevan un rendimiento mayor que las metas ambiguas sólo si los individuos disponen de las competencias que le permiten alcanzar dicha meta.

Hipótesis 2: una instrucción de meta clara producirá mejores resultados que una instrucción de meta ambigua sólo en el caso de que los individuos, aunque no dispongan de competencias aprendidas formalmente, tengan disponible un plan de acción.

Hipótesis 3: Una instrucción de meta ambigua se relaciona positivamente con el logro de meta cuando las personas pueden reformular la meta y establecer un plan de acción. 
Con el objetivo de descartar otras explicaciones plausibles de los potenciales efectos señalados, se consideraron una serie de factores relacionados con la capacidad, la motivación y el proceso de interacción. En concreto, se controlaron factores relacionados con: a) la dificultad de la tarea (capacidad de resolución, experiencia y tiempo de ejecución); b) motivación de los participantes (necesidad de cognición) y c) capacidad de influencia en situaciones de interacción (habilidades sociales). La dificultad de la tarea forma parte del núcleo fuerte de la formulación de la meta (Locke y Latham, 2002), por ello resulta importante controlar el nivel de experiencia previa de los participantes en resolver problemas similares a los que se proponen en los estudios. La ambivalencia se ha asociado en la literatura con un aumento del procesamiento de la información (Briñol et al., 2004). Por lo tanto, la necesidad de cognición, como rasgo, podría tener un efecto en los resultados del presente experimento, toda vez que quienes estén más motivados para pensar podrían hacerlo en mayor medida que el resto, particularmente en la condición de meta ambigua. Por último, la habilidad social se orienta a la consecución de los objetivos en una situación de interacción (Costa y López, 2006). Por lo tanto, cabría esperar que en estos estudios, en los que se afronta una tarea interactiva, las personas que manifiesten un mayor nivel de habilidades social puedan estar en mejores condiciones para conseguir mejores resultados, pudiendo revelar cierto efecto en los resultados de la investigación

Con el objetivo de contrastar los supuestos anteriores se llevaron a cabo tres estudios y un estudio previo (estudio piloto). El estudio previo tenía por objeto garantizar que las instrucciones de meta formuladas por los investigadores se interpretaban en términos de claridad o ambigüedad por personas ajenas a la investigación. En los estudio 1 y 2 (hipótesis 1) analizamos si una meta clara conduce a mejores resultados que una meta ambigua. La diferencia entre ellos estriba en que, mientras en el estudio 1 se contrastó en una muestra de profesionales que participaron en un programa de adquisición de competencias en trabajo en equipo; el estudio 2 examinó si una instrucción de meta clara produce mejores resultados si los participantes carecen de competencias o formación específica para trabajar en equipo. A continuación se realizó un análisis conjunto de ambos estudios. Finalmente, el Estudio 3 exploró el efecto en el logro de meta inducido por la claridad $v s$. ambigüedad en la instrucción de meta cuando los participantes tienen, o no, la posibilidad de planificar sus acciones.

Por tanto, en los estudios 1 y 2 se va a estudiar el efecto de la variable "claridad en la formulación de la meta" en la variable "logro de meta". Además, se analizará el efecto de la variable "competencia en trabajo en equipo" y su interacción con la variable "claridad en la formulación de la meta" en el logro de meta. En el estudio 3 se estudia el efecto de las variables "claridad en la formulación de la meta" y "planificación" en la variable "logro de meta".

\section{Método General}

\section{Estudio piloto}

Con el objetivo de formular las instrucciones de meta clara y ambigua se realizó un estudio piloto. La formulación de las instrucciones de metas clara y ambigua tuvo lugar en dos fases. En primer lugar, tres investigadores redactaron individualmente un total de 24 instrucciones de meta orientados por la concepción de meta clara o ambigua que usamos en la presente investigación (ver más arriba). Posteriormente seleccionaron, por unanimidad, la instrucción que consideraron más clara y la que consideraron con mayor ambigüedad. En segundo lugar, se analizó en qué medida las instrucciones seleccionadas por los investigadores se interpretaban en términos de claridad o ambiguiedad por personas ajenas a la investigación. Un grupo de 27 profesionales con titulación superior, participantes en un curso de formación empresarial sobre habilidades y técnicas de comunicación, cualificaron las dos instrucciones de meta seleccionadas en términos de claridad $v s$. ambigüedad. Tras recibir información sobre los objetivos y las reglas del juego diseñado para los estudios (ver Tabla 1 y Figura 1), respondieron a la siguiente cuestión: Por favor, indique en qué grado la instrucción dada a los participantes para conseguir el objetivo le resultará una meta clara o ambigua en los siguientes casos: "el grupo debe colocar correctamente el mayor número posible de fichas" y "coloque correctamente el mayor número posible de fichas. Se utilizó un formato de respuesta escalar, con puntuaciones comprendidas entre 1 (clara) a 5 (ambigua).

El análisis mediante la prueba $t$ para muestras relacionadas puso de manifiesto que la instrucción " $e l$ grupo debe colocar correctamente el mayor número posible de fichas" tiende a identificarse con una meta clara $(M=1.33 ; S D=0.62)$. Por su parte, la instrucción "coloque correctamente el mayor número posible de fichas" resultó significativamente más ambigua $(M=3.51 ; S D=1.22),[t(26)=-7.35, p<0.001]$. Además, la prueba $t$ para una muestra permitió comprobar cómo la instrucción de meta clara se aleja significativamente del punto medio de la escala (3) $[t(26)=-13.964 ; p<0.001]$; lo mismo se puede decir de la instrucción de meta ambigua pero en dirección al extremo opuesto $[t(26)=2.207 ; p<0.036]$.

Por otro lado, se solicitó a estos colaboradores una predicción sobre qué instrucción resultaría más eficaz para alcanzar la meta, contestaron 25 de los 27 que participaron en esta valoración. El 84\% eligió la instrucción clara "el grupo debe colocar correctamente el mayor número posible de fichas". El resto, $16 \%$, eligió la ambigua: "coloque correctamente el mayor número 
posible de fichas". La prueba de McNemar $(p>0,001)$ indica que resulta significativamente mayor la proporción de sujetos que predijo más eficacia de la instrucción de meta clara.

\section{Variables y Medidas}

Claridad en las instrucciones de meta (estudios 1, 2 y 3). Se manipularon dos condiciones en función de la formulación de la instrucción de meta en términos claros o ambiguos. En la condición de instrucción de meta clara, los participantes ejecutaban el juego con el objetivo: "el grupo debe colocar correctamente el mayor número posible de fichas", indicando explícitamente que la meta para todos y cada uno de los participantes era rellenar el tablero completo. Se entiende por instrucción de meta clara aquella instrucción que no requiere ser interpretada. En la condición de meta ambigua lo ejecutaban con el objetivo: "coloque correctamente el mayor número posible de fichas" que no aclaraba si la meta para cada participante era que el grupo rellenase el tablero completo o que el participante rellenase la subcuadrícula que le había correspondido, dando pie a la interpretación de la meta o a la superposición de metas. También se controló el efecto de la manipulación de la variable en los sujetos que participaron en el experimento. Tras la realización del ejercicio respondieron a la pregunta ¿cómo se ha interpretado la meta a conseguir?, además de inducir una discusión entre los participantes.

Competencias para trabajar en equipo (análisis conjunto de los estudios 1 y 2). Se diferenciaron dos grupos, quienes habían adquirido tales competencias en un curso de formación y quienes no disponían de formación previa en este ámbito. La asignación a estos grupos se realizó en función de la selección de los participantes en los estudios 1 y 2 . Los participantes en el estudio 1 realizaron el experimento como colofón de un curso de formación en trabajo en equipo, mientras que los participantes en el estudio 2 fueron seleccionados con el criterio de no haber recibido formación específica en este campo.

La variable planificación (estudio 3) se manipuló condicionando dos alternativas: proporcionando tiempo para planificar las acciones hacia la consecución de una meta o privando a los participantes de esta posibilidad. Se invitó a los participantes en la condición "con planificación" a realizar la tarea propia de la fase postdecisional del modelo del Rubicón. Dado que el ejercicio imponía las circunstancias temporal -cuándo- y espacial -dónde-, se solicitó planificar únicamente el cómo. Concretamente recibieron la premisa: "Dispone de 10 minutos para planificar cómo va a ejecutar el ejercicio". Los participantes en la condición "sin planificación" realizaron el ejercicio sin opción de pensar en un plan de acción. Disponer o no de tiempo para planificar una tarea es un hecho en sí que no requiere mayor control. No obstante, con intención de garantizar que los participantes en la condición de planificación habían dedicado ciertamente este tiempo a trazar un plan, se les pidió que escribieran las estrategias y el plan de acción diseñado.

Logro de meta. Se consideró el error grupal o número total de errores en el tablero completo como medida de esta variable. El cómputo del número de errores incluía el número de fichas colocadas incorrectamente en el tablero más el número de fichas no colocadas. Se eligió computar el número de errores en lugar del número de aciertos por dos razones: a) el cálculo de los aciertos puede tener distintas interpretaciones [el número total de las casillas del sudoku $\left(81-n^{\circ}\right.$ de errores) $v s$. el número total de casillas que pueden colocar correctamente los participantes $\left(36-\mathrm{n}^{\circ}\right.$ de errores) mientras que el cálculo de los errores no varía en ningún caso; b) la dificultad del juego se va incrementado a medida que se van colocando las fichas, por lo tanto los aciertos que se producen en su inicio no suponen una buena medida de rendimiento.

Variables controladas. Con intención de controlar la capacidad para solucionar el problema se pidió a 16 individuos que lo ejecutasen en un tiempo máximo de 20 minutos, ninguno de ellos participó posteriormente en los experimentos. El 69\% resolvió el juego sin cometer ningún error. El tiempo de ejecución varió entre 6 minutos y los 20 minutos establecidos como tiempo máximo. La experiencia en el juego del Sudoku quedó definida en cuatro categorías ordenadas de menos a más: 1) sin experiencia, 2) con experiencia en Sudokus de nivel fácil, 3) con experiencia en Sudokus de nivel medio y 4) con experiencia en Sudokus de nivel difícil. Además, se estandarizó la variable tiempo, marcando un límite de 40 minutos para la realización del juego en la situación experimental. El límite de 40 minutos se determinó siguiendo un criterio conservador, el doble del tiempo asignado a los 16 colaboradores.

La necesidad de cognición se midió mediante la adaptación española de la Escala de Necesidad de Cognición de Cacioppo y Petty (Falces, Briñol, Sierra, Becerra y Alier, 2001). La versión española de la escala presenta propiedades psicométricas que acreditan su uso (alfa de Cronbach $=0.87$ ). La escala está compuesta por 15 ítems con un formato de respuesta cuyas puntuaciones varían entre 1 (nada característica de mî) y 5 (muy característica de mî).

Las habilidades sociales se midieron mediante el Inventario de Asertividad de Rathus (1973) compuesto por 30 ítems con respuesta escalar de 1 a 6 donde 1 significa "Muy característico en mî" y 6 "Muy raro en mî". Nevid y Rathus (1979) encontraron un coeficiente de fiabilidad adecuado utilizando el método de dos mitades $(r=0.77, p=0.01)$. Recientemente, en una muestra japonesa (Suzuki, Kanoya, Katsuki y Sato, 2007) el inventario presentó garantías psicométricas que siguen avalan su fiabilidad (alfa de Cronbach $=0.84$ ). 


\section{Procedimiento}

Cada uno de los tres experimentos se presentó a los participantes como dos tareas distintas. En el primer lugar se solicitó a los participantes contestar a la escala de necesidad de cognición y al inventario de asertividad (ver variables controladas) bajo el pretexto de baremarlos. Seguidamente, se comenzó el experimento asignando aleatoriamente a los participantes a las condiciones experimentales. El colaborador que monitorizó el experimento desconocía los objetivos e hipótesis de la investigación (procedimiento del doble ciego destinado a prevenir los efectos no intencionados del experimentador en la conducta de los participantes).

La tarea se diseñó atendiendo a cuatro características que delimitaban la situación experimental, tras recibir la instrucción de meta: a) los participantes determinan qué conseguir y cómo conseguirlo, interpretación; b) actúan teniendo en cuanta las acciones de los demás, interacción; c) obtienen resultados que dependen en alguna manera de los otros, interdependencia y d) disponen de los recursos necesarios y suficientes para lograr el objetivo, adecuación de recursos. Con estas características se pretende garantizar que la situación experimental disfruta de las condiciones propias de una dinámica grupal. Además, se evitaría un conflicto potencial por la insuficiencia de recursos. La tarea consistió en resolver un Sudoku en grupo (cuatro personas), donde a cada participante se le asignó aleatoriamente una de las cuatro subcuadrículas que ocupan las esquinas del tablero y que tendrían que rellenar conforme a las reglas del Sudoku (es decir, cada participante contará con un sobre con 9 fichas -del 1 al 9-; deberá colocar las fichas en la subcuadrícula de $3 \times 3$ que le toque por sorteo; colocará sus fichas respetando la regla del Sudoku: no puede repetirse ninguna cifra en ninguna fila, columna o subcuadrícula de $3 \times 3$ ). Los números de la casilla central no se especificaron porque no tienen incidencia en el juego y facilitan la visibilidad de las diferentes subcuadrículas Las otras cuatro subcuadrículas se presentan completamente rellenas (ver Figura 1). Para la ejecución del ejercicio se utilizó el
Sudoku de Rubik ${ }^{\circledR}$. Este tablero presenta unas particularidades que lo hacen especialmente útil (p.ej., las fichas con los dígitos del 1 al 9 están estandarizadas por lo que se evita cualquier error o distorsión debido a la escritura propia de cada individuo. Cada dígito está impreso en un color distinto facilitando visualmente la tarea. Cada participante puede recibir en un sobre las fichas que posteriormente ha de colocar en el tablero).

Figura 1. Tablero del Sudoku al inicio del experimento

\begin{tabular}{|l|l|l|l|l|l|l|l|l|}
\hline & & & 2 & 1 & 9 & & & \\
\hline & & & 8 & 7 & 3 & & & \\
\hline & & & 4 & 6 & 5 & & & \\
\hline 7 & 8 & 6 & & & & 3 & 4 & 2 \\
\hline 2 & 5 & 9 & & & & 8 & 1 & 6 \\
\hline 4 & 1 & 3 & & & & 5 & 9 & 7 \\
\hline & & & 7 & 2 & 4 & & & \\
\hline & & & 5 & 3 & 8 & & & \\
\hline & & & 1 & 9 & 6 & & & \\
\hline
\end{tabular}

Antes de empezar el juego, los participantes respondieron a cuestiones sobre su experiencia en el Sudoku y fueron informados de las reglas especiales del juego (ver Tabla 1). Concluido el ejercicio, el colaborador registró el número de errores de cada grupo. A continuación, se controló la manipulación experimental y se realizó una discusión en grupo donde los participantes expresaron sus impresiones sobre el experimento y manifestaron explicaciones que interpretaban los resultados. Finalmente, el experimentador les reveló las características y el objetivo del estudio (debriefing).

\section{Análisis de datos}

Para el estudio 1, considerando el tamaño de la muestra $(n=16)$, se utilizó una prueba no paramétrica para dos muestras independientes (Mann-Whitney),

Tabla 1. Instrucciones del juego

1. El juego tiene lugar sobre un tablero de Sudoku situada en frente de los 4 jugadores. Los 4 jugadores permanecen sentados excepto en el momento que les toque jugar.

2. Cada participante contará con un sobre con 9 fichas (del 1 al 9). Las fichas deben ser colocadas en la subcuadrícula de 3x3 que le toque por sorteo.

3. El juego se realiza siguiendo una secuencia de turnos. 9 en total. Comenzará un jugador seleccionado al azar. En el siguiente turno lo iniciará el situado a la derecha.

4. En cada turno cada jugador colocará una de sus fichas respetando la regla del Sudoku: no puede repetirse ninguna cifra en ninguna fila, columna o subcuadrícula de $3 \times 3$

4. La ficha una vez colocada no se podrá retirar del tablero.

6. Cada jugador dispondrá de 1 minuto para colocar su ficha, pasado este tiempo perderá su turno.

7. El juego termina al concluir los 9 turnos. 
menos exigente en sus supuestos. En el estudio 2, se utilizo la prueba $\mathrm{T}$ para muestras independientes. Sin embargo, debemos tener en cuenta que hay variables que pueden estar afectando al rendimiento en la tarea, como son la experiencia previa con tareas de sudoku, la necesidad de cognición y las habilidades sociales. Puesto que el diseño no permitió controlar estas variables a priori, se realizó un control estadístico del efecto de estas variables. El procedimiento de análisis de covarianza (ANCOVA) utiliza como variable dependiente los errores de los pronósticos resultantes de un análisis de regresión múltiple con las variables que van a ser controladas. Por ello, en los tres estudios se utilizó el procedimiento de análisis de covarianzas. Puesto que este procedimiento requiere el cumplimiento de los mismos supuestos que el ANOVA paramétrico, se realizaron pruebas de normalidad (Kolmogorov-Smirnov) y homocedasticidad (Levene) sobre los datos obtenidos. Por otro lado, en caso de utilizar un diseño factorial intersujetos $2 \times 2$ y cuando el efecto de las dos variables independientes fue menor que el efecto de la interacción se informó de los efectos simples por separado (León y Montero, 2001). Las comparaciones de efectos simples para analizar la interacción se realizaron siguiendo el procedimiento descrito por Pardo, Garrido, Ruiz y San Martín (2007). Por último, cabe señalar que cuando se utilizan escalas de medida con diferentes rangos de respuesta conviene utilizar puntuaciones $z$. En los estudios que presentamos no se ha procedido a tipificar las puntuaciones ya que no se realizan comparaciones entre las variables. Es más, los análisis de covarianza, donde se incluyen dos VI's (Factores) y una serie de variables (covariables) que se desea controlar debido a sus potenciales efectos en la VD, se ejecutan a partir de los residuos de un análisis de regresión donde no es necesario que las covariables estén en la misma métrica, luego las escalas de medida pueden ser diferentes.

\section{ESTUDIO 1}

En este estudio se sometió a prueba si una instrucción de meta clara produce mejores resultados que una intención o instrucción de meta ambigua. Con ello se pretendía contractar, de forma exploratoria, los resultados de las investigaciones previas.

\section{Método}

\section{Participantes}

Participaron voluntariamente 16 graduados universitarios (ocho eran hombres y ocho mujeres) de distintas titulaciones que desarrollaban su actividad profesional en diversas empresas. Los participantes estaban realizando un curso de adquisición de competencias para "trabajar en equipo" en el marco del programa CITIUS de iniciación profesional en la empresa. El curso tuvo una duración de 20 horas y dedicaron las dos últimas horas para realizar la tarea. La edad de las participantes estuvo comprendida entre los 24 y 27 años $(M=24.94 ; S D=0.93)$.

\section{Diseño}

Se utilizo un diseño factorial intersujetos con la claridad en la formulación de meta (instrucción de meta clara $v s$. instrucción de meta ambigua) como variable independiente y el logro de meta como variable dependiente.

\section{Resultados}

\section{Variable dependiente}

La prueba de Mann-Whitney resultó significativa, indicando que existen diferencias en cuanto al rendimiento (número de errores) entre el grupo que recibió una instrucción de meta clara y el grupo que con instrucción de meta ambigua $(U=0.000 ; p<0.005)$. El procedimiento de análisis de covarianza permitió descartar el efecto de las variables necesidad de cognición $[F(1,11)=4.430 ; p=0.059] \mathrm{y}$ habilidades sociales $[F(1,11)=0.616 ; p=0.449]$. Sin embargo, se observó que la variable experiencia sí tiene efecto sobre los pronósticos de la variable dependiente, por lo que debe ser controlada $[F(1,11)=10.900 ; p=0.007]$.

La prueba ANCOVA, después de eliminar el efecto de las covariables, mostró un efecto de la claridad de las instrucciones de metas sobre el rendimiento del grupo, de tal forma que los grupos asignados a la condición de instrucción de meta clara mostraron menor número de errores (ver tabla 2), es decir, mejor logro de la meta $(M=2.00 ; S D=0.00)$ que aquellos grupos que recibieron la instrucción de meta ambigua $(M=7.00 ; S D=1.06),[F(1,14)=303.643, p<0.001]$, Además, encontramos que el tamaño del efecto resultó relevante. El 96,9\% de la variabilidad de los errores cometidos por los participantes en la prueba se puede interpretar por la condición experimental a que se sometió a los participantes $\left(\eta^{2}=0.965\right)$.

\section{Control de la manipulación post-experimental}

Tanto mediante el control de la manipulación experimental como en la discusión posterior al experimento, el patrón de respuestas de los participantes bajo la condición de meta clara mostró una coincidencia en la interpretación de la instrucción como el logro de la meta por el equipo. Las respuestas de los participantes 
Tabla 2. Estadísticos descriptivos del logro de meta en los tres estudios

\begin{tabular}{|c|c|c|c|c|}
\hline & Condición & $\mathrm{N}$ & $\mathrm{M}^{*}$ & SD \\
\hline $\begin{array}{l}\text { Experimento } 1 \\
\text { (con formación en competencias) }\end{array}$ & $\begin{array}{l}\text { Meta clara } \\
\text { Meta ambigua }\end{array}$ & $\begin{array}{l}8 \\
8\end{array}$ & $\begin{array}{l}2.00 \\
7.00\end{array}$ & $\begin{array}{l}0.00 \\
0.00\end{array}$ \\
\hline $\begin{array}{l}\text { Experimento } 2 \\
\text { (sin formación en competencias) }\end{array}$ & $\begin{array}{l}\text { Meta clara } \\
\text { Meta ambigua }\end{array}$ & $\begin{array}{l}40 \\
40\end{array}$ & $\begin{array}{l}7.30 \\
5.60\end{array}$ & $\begin{array}{l}2.83 \\
2.57\end{array}$ \\
\hline $\begin{array}{l}\text { Análisis conjunto } \\
\text { (Experimentos } 1 \text { y } 2 \text { ) }\end{array}$ & $\begin{array}{l}\text { Meta clara } \\
\text { Meta ambigua } \\
\text { Con formación en competencias } \\
\text { Sin formación en competencias }\end{array}$ & $\begin{array}{l}48 \\
48 \\
16 \\
80\end{array}$ & $\begin{array}{l}6.42 \\
5.83 \\
4.50 \\
6.45\end{array}$ & $\begin{array}{l}3.26 \\
2.43 \\
2.68 \\
2.82\end{array}$ \\
\hline Experimento 2 & $\begin{array}{l}\text { Meta clara } \\
\text { Meta ambigua } \\
\text { Con planificación } \\
\text { Sin planificación } \\
\text { Meta clara * con planificación } \\
\text { Meta clara * sin planificación } \\
\text { Meta ambigua * son planificación } \\
\text { Meta ambigua * sin planificación }\end{array}$ & $\begin{array}{l}32 \\
32 \\
32 \\
32 \\
16 \\
16 \\
16 \\
16\end{array}$ & $\begin{array}{l}6.75 \\
4.25 \\
3.63 \\
7.38 \\
4.25 \\
9.25 \\
3.00 \\
5.50\end{array}$ & $\begin{array}{l}3.65 \\
2.58 \\
3.05 \\
2.59 \\
2.96 \\
2.35 \\
3.10 \\
0.89\end{array}$ \\
\hline
\end{tabular}

Nota. ${ }^{*}=$ Numero de errores

bajo la condición de meta ambigua presentaron mayor variabilidad y nos dan pistas de que una instrucción de meta resulta ambigua puede serlo en un doble sentido: a) es susceptible de interpretación o b) se identifica individualmente como una meta clara pero entra en conflicto con la interpretación de los otros miembros del grupo.

El segundo objetivo de esta discusión fue explorar posibles variables no controladas en el diseño del experimento y conocer las explicaciones de los participantes sobre los resultados. Los participantes atribuyeron los resultados a la asociación que habían realizado con los contenidos y experiencias del curso en el que estaban participando. Quienes se enfrentaron a la instrucción de meta clara afrontaron la experiencia como una actividad más de las realizadas en el curso. Es decir, la instrucción que contenía la palabra "grupo" la encontraron coherente con el desarrollo de la acción formativa. Por el contrario, quienes participaron en la condición meta ambigua indicaron que se sintieron desubicados, incluso engañados, al percibir una situación incoherente entre la instrucción del ejercicio y la expectativa de una actividad grupal propia del curso. Según manifestaron esta situación incoherente determinó una percepción de falta de control de la situación.

\section{Discusión}

Los resultados son consistentes con la importancia asignada a la claridad de la meta en las investigaciones previas. En nuestro estudio los resultados verifican que la claridad de metas presenta un efecto positivo en el logro de la meta. Pero, la aportación relevante del estudio no fue tanto la verificación de este efecto sino la detección de diversas condiciones que hipotéticamente pueden modularlo. Los participantes bajo la condición de meta ambigua atribuyeron sus peores resultados no tanto a la ambigüedad de la meta sino a la disonancia entre la meta y las estratégicas, técnicas y procedimientos para la solución de problemas (competencias) en grupo que habían aprendido. Es decir, imputaron los resultados no al qué sino al cómo o mejor, a la ambivalencia en la interacción entre el qué y el cómo.

Aunque los resultados obtenidos confirman la idea de que la claridad de las instrucciones de meta provoca mejores resultados, resulta plausible pensar que la coherencia entre la claridad de la meta -saber qué- y las estrategias disponibles -saber cómo- es potencialmente responsable de los resultados. Por un lado, los procedimientos de acción aprendidos en la acción formativa se activarían mediante el heurístico de accesibilidad (Kahneman, 2003; Taylor, 1982; Tversky y Kahneman, 1974). Por otro, los participantes en la condición de meta ambigua deberían resolver la discrepancia (p.e. Abelson et al., 1968; Festinger, 1957) entre las estrategias accesibles y una meta que resultaría incoherente con el uso de tales estrategias. Por ello, planteamos si la claridad de las instrucciones de meta habría influido en los resultados en combinación a los esquemas de acción suscitados (Kruglanski, 1989, 1996; Kruglanski y Klark, 1985) que actuarían de mediadores. De hecho, Latham y Locke (2007) añaden la capacidad a la lista de moduladores que influyen en los efectos del contenido de la tarea.

En resumen, aunque los resultados del experimento verificaron la hipótesis de la importancia de la claridad 
de las instrucciones de meta para su logro, la condición manipulada no parece suficiente para explicar el efecto observado. Por ello, se planteó un segundo experimento donde los participantes no tuvieran disponible una formación específica. Esto es, no dispusieran de estrategias aprendidas formalmente sobre la solución de ejercicios grupales.

\section{ESTUDIO 2}

En este experimento se volvió a someter a prueba si una instrucción de meta clara produce mejores resultados que una instrucción de meta ambigua, pero controlando el factor que pudo influir en los resultados del estudio 1. Es decir, en este experimento, los sujetos quedan expuestos a la situación sin unas estrategias de solución formadas previamente. (i.e., curso de formación en competencias para trabajar en equipo). Además, se ejecutó un análisis conjunto de los experimentos 1 y 2 . Este análisis se fundamenta en la importancia teórica atribuida a la relación entre la claridad de la meta y las competencias para solucionar el ejercicio.

\section{Método}

\section{Participantes}

Participaron voluntariamente 80 estudiantes universitarios de distintas titulaciones de la Universidad Autónoma de Madrid. La edad de las participantes estaba comprendida entre los 18 y 49 años $(M=21.85 ; S D$ $=4.44)$. De los cuales, 14 fueron hombres $(17.5 \%)$ y 66 mujeres $(82.5 \%)$. Ninguno de ellos había tenido formación específica en "trabajo en equipo". La decisión de seleccionar estudiantes universitarios para la realización del experimento estaba motivada por el supuesto de que estos tendrían, además, menos experiencia en trabajar en equipo que las personas en activo.

A diferencia del experimento 1, en este estudio los participantes: a) se reclutaron en el Campus de la universidad individuos que no habían recibido ni estaban recibiendo entrenamiento en trabajo en equipo o similar; b) la asignación del grupo a la condición experimental se realizó previamente, manteniendo la aleatoriedad c) en la asignación de sujetos a grupos se controló que los participantes no se conociesen.

\section{Diseño}

El diseño fue similar al seguido en el experimento 1. Posteriormente, en el análisis conjunto de los experimentos 1 y $2(n=96)$, se utilizó un diseño factorial intersujetos $2 \times 2$ considerando dos variables independientes: una variable manipulada experimentalmente (instrucción de meta clara $v s$. instrucción de meta ambigua) y una segunda variable independiente $e x$ post facto (con formación previa en competencias para trabajar en equipo $v s$. sin formación previa en competencias para trabajar en equipo).

\section{Resultados}

\section{Variable dependiente}

La prueba t para muestras independientes mostró un efecto estadísticamente significativo de la claridad de las metas sobre los resultados pero en sentido inverso al observado en el experimento 1 (ver tabla 2). Los participantes asignados a la condición de instrucción de meta clara mostraron mayor número de errores $(M=7.30 ; S D=2.83)$; es decir, peor rendimiento que los participantes en la condición de instrucción de meta ambigua $(M=5.60 ; S D=2.57),[t(78)=2.812$, $p=0.006]$.

Con intención de controlar el posible efecto de otras variables que pudieran estar afectando a los resultados se procedió a ejecutar un análisis de covarianza tal y como se hiciera en el estudio 1. En este caso, ninguna de ellas mostró efecto significativo en el logro de la meta: experiencia en el sudoku $[F(1,75)=0.320$; $p=0.573]$; necesidad de cognición $[F(1,75)=0.020$; $p=0.887]$ y habilidades sociales $[F(1,75)=0.015$; $p=0.901]$. Una vez controlada la influencia de estas covariables, los resultados del análisis constataron el efecto de las instrucciones de meta en el logro de meta $\left[F(1,75)=51.533 ; p=0.011 ; \eta^{2}=0.083\right]$. No obstante, es necesario atender a la escasa variabilidad del resultado que el factor manipulado parece explicar, únicamente el $8.3 \%$.

\section{Análisis conjunto de los Experimentos 1 y 2}

Los datos de este análisis deben considerarse con las consiguientes cautelas. Por un lado, conviene recordar el reducido tamaño de la muestra en la condición formación. Por otro, es necesario señalar que la participación en las condiciones de la variable formación no fue aleatoria. En línea con los resultados de ambos experimentos, el análisis de covarianzas 2 x 2 reveló un efecto significativo de la instrucción de meta $[F(1.89)=$ 5.751; $\left.p=0.019 ; \eta^{2}=0.061\right]$. Quienes resolvieron el ejercicio bajo la condición de meta clara cometieron más errores que aquellos que lo hicieron desde la instrucción de meta ambigua $(M=6.42 ; S D=3.26 v s$. $M=$ $5.83 ; S D=2.43$ ). Tal y como se preveía, el haber recibido o no, formación en trabajo en equipo reveló diferencias significativas entre los grupos. Los participantes en la condición "formación" rindieron mejor $(M=$ 4.50; $S D=2.68$ ) que los que no habían participado de una acción específica en trabajo en equipo $(M=6.45$; $\left.S D=2.82), F(1,89)=7.984 ; p=0.006 ; \eta^{2}=0.082\right]$ 
También se puso de manifiesto el efecto significativo de la interacción $[F(1,89)=23.240 ; p<0.001$; $\left.\eta^{2}=0.207\right]$ (ver Figura 2). Las comparaciones de efectos simples para analizar la interacción revelaron diferencias entre las condiciones formación y "sin formación" ( $M=2 ; S D=0.00$ vs. $M=7.30 ; S D=2.83)$ cuando los participantes recibieron la instrucción de meta clara $(d=-5.348 ; E T=0.987 ; p<0.001)$. Por el contrario, cuando se comparan los resultados de los individuos en la condición de meta ambigua no se hallaron diferencias entre quienes recibieron formación $(M=7 ; S D=$ $1.07)$ y $\operatorname{los}$ que no $(M=5.6 ; S D=2.57),(d=1.362$; $E T=0.995 ; p=0.174)$. No se presenta los efectos simples de las condiciones de formación dado que los resultados son coincidentes con los expuestos en los experimentos 1 -con formación- y 2 -sin formaciónpor separado.

Ninguna de las covariables mostró una influencia significativa en los resultados del grupo: experiencia en el sudoku $[F(1,89)=0.713 ; p=0.401]$; necesidad de cognición $[F(1,89)=0.096 ; p=0.758]$ y habilidades sociales $[F(1,89)=0.037 ; p=0.847]$.

\section{Discusión}

Los resultados de este estudio muestran cómo una instrucción de meta clara no ejerce, por sí misma, un efecto positivo sobre el logro de la meta. Al contrario, en la situación experimental propuesta, quienes ejecutaron la tarea bajo la instrucción de meta ambigua obtuvieron mejores resultados. La ausencia de entrenamiento en estrategias para trabajar en equipo ha puesto de manifiesto dos incógnitas que necesitan respuesta: ¿Por qué se anula el efecto positivo de la claridad en la instrucción de meta sobre el rendimiento hallado en las investigaciones anteriores?, ¿Por qué la instrucción de meta ambigua lleva a unos resultados más exitosos en contra de toda predicción?

Por otra parte, el análisis conjunto de los experimentos 1 y 2 informó de un efecto de la interacción sobre los resultados. Los resultados del análisis indican que bajo la condición de formación, la instrucción de meta ambigua hace aumentar el número de errores (Exp. 1). En cambio, bajo la condición "sin formación" es la instrucción de meta clara lo que provoca el aumento del error (Exp. 2). Además, se ha encontrado que los individuos que reciben una instrucción de meta clara ven afectado su rendimiento por la formación previa: obtienen resultados más favorables los individuos entrenados. Por su parte, los participantes que reciben una instrucción de meta ambigua no ven influenciado significativamente los resultados de su trabajo por el hecho de haber sido formados. Por lo tanto, habría que añadir una tercera cuestión a las dos anteriores ¿Por qué disponer o no disponer de formación específica previa no resulta relevante cuando los individuos reciben una instrucción de meta ambigua?

Figura 2. Logro de meta en función de la instrucción de meta y la formación

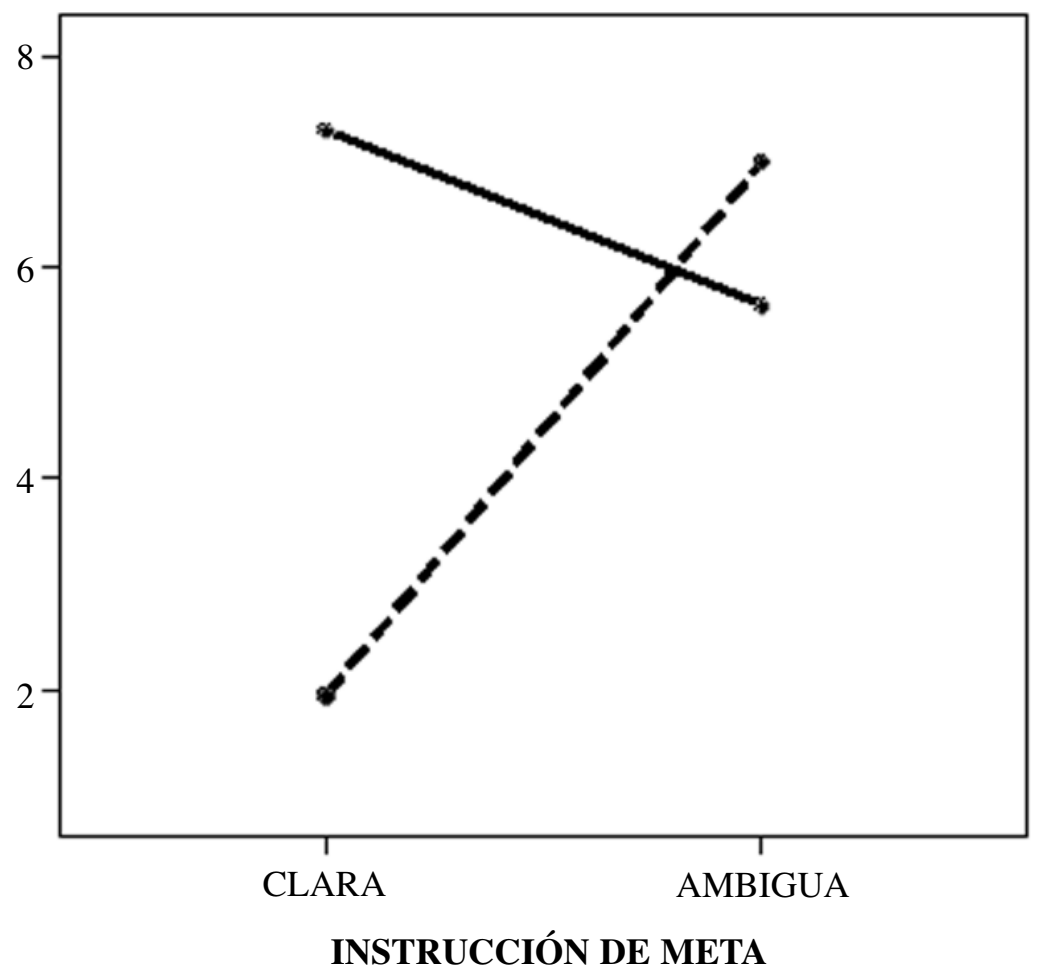

CON FORMACIÓN

- - CON FORMACIÓN

- SIN FORMACIÓN 
¿Por qué se anula el efecto positivo de la claridad en la instrucción de meta sobre el rendimiento hallado en las investigaciones anteriores? Metas claras son aquellas que especifican un estado final deseado que no necesita ser interpretado. Por lo tanto, estimulan el inicio de la acción de forma automática e inmediata. Los participantes en la condición de instrucción de meta clara recibieron una formulación de meta que especificaba el estado final deseado. Entonces, iniciaron la acción de forma inmediata y automática. Ahora bien, cuando los participantes no dispusieron de estrategias adecuadas para la solución de un problema grupal, el hecho de pasar a la acción de forma automática no condujo al éxito. Por lo tanto, se advierte una situación de ambivalencia al tener clara la meta -saber qué- pero no disponer de estrategias adecuadas - no saber cómo-. La falta de ajuste entre las demandas de la tarea y los recursos disponibles por el individuo para dar una respuesta adecuada ha sido ampliamente estudiada en el contexto organizacional. Concretamente, diversos modelos explicativos de la tensión y el estrés se describen en términos comparativos entre la demanda y los recursos (p.e. Karasek, 1979; McGrath, 1976). Un estudio reciente muestra cómo los sujetos sometidos a una condición de alta demanda y bajo control ven mermada su capacidad para procesar información (De Miguel et al., 2009). Desconocemos si esta situación de ambivalencia produjo tensión en los participantes del estudio. No obstante, la investigación previa advierte que tal incoherencia perjudica al rendimiento (Peiró y Salvador, 1993).

¿Por qué la instrucción de meta ambigua lleva a unos resultados más exitosos en contra de toda predicción? Por su parte, las metas ambiguas se han conceptualizado como aquellas que requieren dan lugar a diferentes interpretaciones. Por lo tanto requieren de una elaboración posterior. En este caso, encontramos una situación en donde los sujetos no tienen claro el objetivo a conseguir - no saber qué- y tampoco tienen claro qué estrategias deben utilizar para solucionar el problema -no saber cómo-. Dada la condición ambigua de la tarea, la solución del problema requiere, en primer lugar, definir la meta. De este modo, al no tener disponibles estrategias previas, la definición de la meta no entra en conflicto con ningún curso de acción estandarizado. Es más, hipotéticamente los individuos ajustan la meta con el curso de acción que ellos mismos diseñan posteriormente (Heckhausen y Gollwitzer, 1987).

¿Por qué disponer o no disponer de formación específica previa no resulta relevante cuando los individuos reciben una instrucción de meta ambigua? En línea con la explicación anterior, la primera operación que realizan los individuos que reciben una instrucción de meta ambigua consiste en interpretar la meta para definirla. Una vez que han redefinido su meta, conciben un plan de acción donde, eso sí, integrarán los cursos de acción que previamente les han resultado exitosos (Gollwitzer, 1996).
Esta explicación tentativa, que vincula el éxito de logro a la coherencia o ambivalencia entre la claridad de la meta y los cursos de acción, abre una línea interpretativa que se debe contrastar. Con intención de ponerla a prueba, realizamos un tercer experimento.

\section{ESTUDIO 3}

La incorporación del plan de acción como factor hipotéticamente determinante del logro de la meta obliga a reconsiderar los modelos explicativos que abunden, además de en la formulación de metas, en los esfuerzos para conseguirlas. En este experimento se contrastan las hipótesis 2: una instrucción de meta clara producirá mejores resultados que una instrucción de meta ambigua sólo en el caso de que esté disponible un plan de acción y 3: una instrucción de meta ambigua se relaciona positivamente con el logro de meta cuando las personas pueden reformular la meta y establecer un plan de acción.

\section{Método}

\section{Participantes}

Participaron voluntariamente 64 estudiantes universitarios de distintas titulaciones de la Universidad Autónoma de Madrid que realizaron el experimento de forma voluntaria (50 mujeres y 14 hombres). Su rango de edad estaba comprendido entre los 18 y 32 años $(M=22.06 ; S D=3.24)$. Las características de los participantes coincidieron, en cuanto a la ausencia de formación previa en estrategias para trabajar en equipo, con las comentadas en el experimento 2 .

\section{Diseño}

Se utilizó un diseño factorial intersujetos 2 (Instrucción de meta clara $v s$. instrucción de meta ambigua) $x 2$ (con planificación $v s$. sin planificación). Al igual que en los estudios anteriores se controló la experiencia previa en realizar Sudokus, la necesidad de cognición y las habilidades sociales.

\section{Resultados}

El análisis de covarianza reveló un efecto principal de la claridad de las instrucciones de meta, $[F(1,57)=$ 16.896; $\left.p<0.001 ; \eta^{2}=0.229\right]$. Los participantes asignados a la condición de instrucción de meta clara mostraron mayor número de errores, es decir menor éxito en el logro de la meta, que los participantes en la condición de instrucción de meta ambigua $(M=6.75 ; S D=$ 
3.65 vs. $M=4.25 ; S D=2.58$ ), (ver Tabla 2). Como se había previsto, el efecto principal de la planificación resultó también significativo, $[F(1,57)=34.847$; $\left.p<0.001 ; \eta^{2}=0.379\right]$, mostrando diferencias entre quienes pudieron planificar $(M=3.63 ; S D=3.05)$ y los que no $(M=7.38 ; S D=2.59)$.

Los resultados revelaron, además, un efecto de la interacción entre la instrucción de meta y la planificación $\left[F(1,57)=31.209 ; p=0.030 ; \eta^{2}=0.080\right]$ (Ver Figura 3). Los contrastes mostraron que en las dos condiciones de instrucción de meta, fueron más exitosos los participantes que tuvieron ocasión de planificar. Ante la instrucción de meta clara, los participantes cometieron significativamente menos errores planificando $(M=4.25 ; S D=2.96)$ que sin planificar $(M=$ 9.25; $S D=2.35),(d=-5.180 ; E T=0.903 ; p<0.001)$. Lo mismo ocurrió ante la meta ambigua entre quien dispuso de tiempo para diseñar un plan y quien no tuvo esta oportunidad $(M=3.00 ; S D=3.10$ y. $M=5.50 ; S D=0.89$ respectivamente $),(d=-2.262 ; E T=0.918 ; p=0.017) . \mathrm{La}$ comparación entre la instrucción de meta clara y la ambigua mostró diferencias significativas cuando se analizan entre los participantes en la condición "sin planificación" ( $d=4.225 ; E T=0.983 ; p<0.001)$, en cambio estas diferencias no resultaron significativas entre los sujetos que tuvieron ocasión de planificar $(d=1.306 ; E T=0.895 ; p=0.150)$.

Las covariables no revelaron efectos significativos en los resultados del grupo: experiencia en el sudoku $[F(1,57)=0.848 ; p=0.361]$; necesidad de cognición
$[F(1,57)=0.329 ; p=0.568]$ y habilidades sociales $[F(1,57)=0.656 ; p=0.421]$.

\section{Discusión}

De nuevo, se ha observado cómo una instrucción de meta clara no garantiza el éxito en el logro de la meta. Además, se reitera que la instrucción de meta ambigua ha derivado hacia resultados más exitosos que la instrucción de meta clara. Concretamente, los resultados hallados entre los individuos que no tuvieron ocasión de planificar replican los encontramos en el segundo experimento. Es decir, la meta ambigua llevó a mejores resultados. La inclusión de la variable planificación reveló nuevas conclusiones: a) la imposibilidad de planificar anula el efecto positivo de la claridad de la meta; b) la posibilidad de planificar anula el efecto de la instrucción de meta. Es más, muestra un efecto positivo en el rendimiento con independencia de la meta formulada previamente por el experimentador; c) La mejor combinación es aquella que permite al sujeto pensar y decidir $q u e ́$ meta conseguir junto al diseño de un plan de acción, cómo conseguirlo (meta ambigua con planificación) y d) La peor combinación es aquello que impone la meta (p.e., no da pie a interpretarla, derogando la decisión del individuo) y no permite diseñar cauces de acción para lograrla (meta clara sin planificación).

Estas conclusiones apuntan que las personas que pudieron procesar las tareas de las dos primeras fases

Figura 3. Logro de meta en función de la instrucción de meta y la planificación

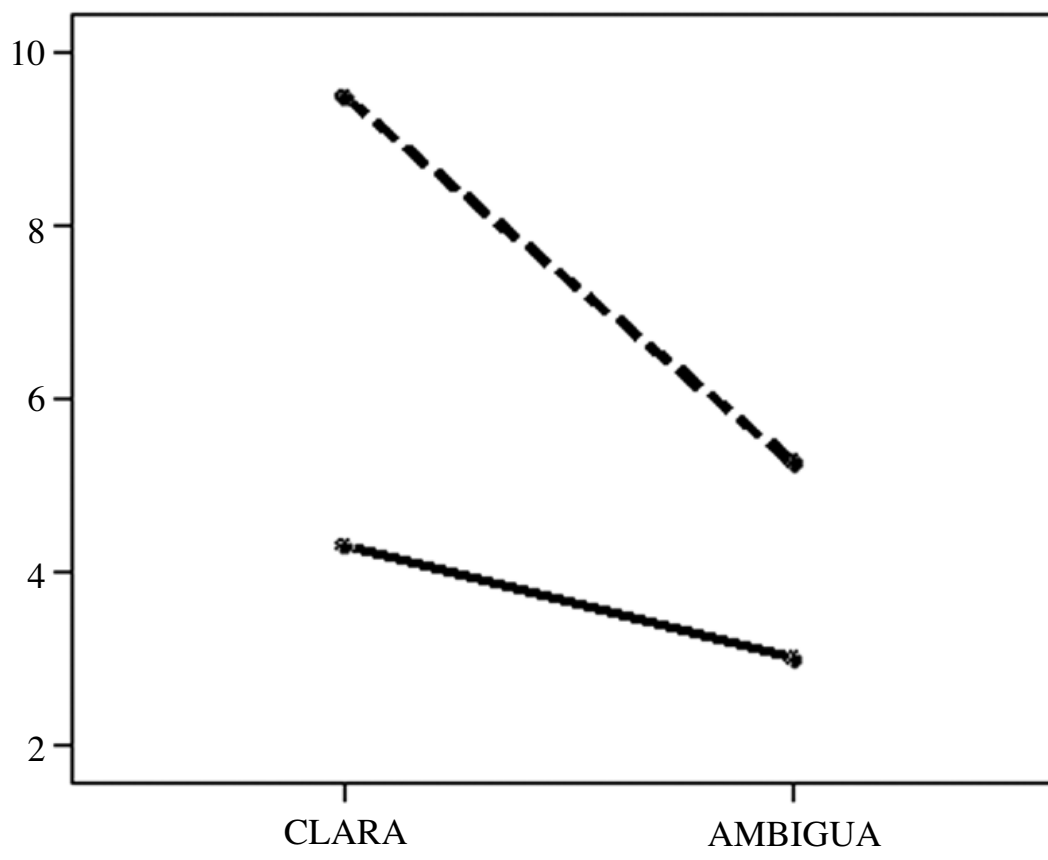
PLANIFICACIÓN
- - SIN PLANIFICACIÓN
- CON PLANIFICACIÓN

INSTRUCCIÓN DE META 
del Modelo del Rubicón pasaron a la acción (la tercera fase) sin problema, implementando la meta y obteniendo mejores resultados (Heckhausen y Gollwitzer, 1987; Gollwitzer, 1990,1996). Por otro lado, inducidos los individuos a diseñar un plan de acción en pro de una meta, parece sensato pensar que la interpretación de la meta fue la primera operación inherente al proceso de logro. Toda vez que no se pude concebir un plan de acción sin definir previamente el objetivo. En otras palabras, los individuos decidieron el qué y el cómo. Esta secuencia daría cuanta de las razones que explican el efecto del plan con independencia de la instrucción de meta y resultan acordes con este modelo teórico y los resultados empíricos del estudio.

\section{Discusión General}

La presente investigación analiza diversos efectos de la formulación de las metas y los esfuerzos que el individuo realiza para conseguirlas (goal setting y goal striving). Los estudios 1 y 2 se centran en la formulación de metas, específicamente en la claridad o ambigüedad con que estas se formulan. El estudio 3 combina la formulación de metas con los procesos de planificación. Concretamente la claridad o ambigüedad de las metas junto a la posibilidad de diseñar un plan de acción o no poder hacerlo. Los resultados de estos experimentos apuntan a una confirmación de las hipótesis 1 y 3: "las metas claras conllevan un rendimiento mayor que las metas ambiguas sólo si los individuos disponen de las competencias que le permiten alcanzar dicha meta" y "una instrucción de meta ambigua se relaciona positivamente con el logro de meta cuando las personas pueden reformular la meta y establecer un plan de acción", respectivamente. No ocurre lo mismo con la hipótesis 2: "una instrucción de meta clara producirá mejores resultados que una instrucción de meta ambigua sólo en el caso de que los individuos, aunque no dispongan de competencias aprendidas formalmente, tengan disponible un plan de acción". Hemos encontrado que la posibilidad de planificar anula el efecto de la claridad o ambigüedad en la formulación de meta sobre el logro de la misma.

Consideramos que los resultados de esta investigación son relevantes por tres razones: a) complementan la premisa de partida, poniendo de manifiesto cómo la claridad en la meta no siempre conduce de forma inmediata al éxito; b) amplia el conocimiento sobre los efectos de la formulación de las metas en el logro, ya que, como hemos expuesto, la ambigüedad en la meta puede ser más exitosa que la claridad en ciertas circunstancias y c) clarifica ciertas relaciones entre la formulación de metas y los esfuerzos para lograrlas. En este último sentido, se ha mostrado como la claridad en la meta es exitosa siempre y cuando sea consistente con la posesión de estrategias específicas para afrontarla. Por el contrario, la ambigüiedad en la meta es más exitosa cuando no se dispone de estrategias de afrontamiento o de un plan de acción. Además, es importante señalar el efecto favorable que presenta la planificación sobre el logro de metas con independencia de cómo estén formuladas. De hecho, en esta investigación el plan de acción inhibe el efecto de la formulación de metas.

Estos resultados se pueden explicar atendiendo a procesos psicólogos de carácter motivacional y volitivo. La conducta de las personas se dirige a encontrar un estado de coherencia interna y de equilibrio con su entorno. De ahí que planifiquen sus conductas con el fin de obtener consecuencias deseables y conseguir unos objetivos y fines planeados. Una formulación de meta clara es exitosa cuando el individuo dispone de estrategias adecuadas. En este caso, la necesidad de coherencia está cubierta y las personas pueden pasar directamente a la acción, sin necesidad de elaborar explícitamente un plan. De hecho, en la fase accional se ejecuta la tarea de forma deliberada o automática (Bargh y Gollwitzer, 1994; Oettingen, Grant, Smith, Skinner y Gollwitzer, 2007).

Ahora bien, de aparecer discrepancia, la búsqueda de equilibrio y coherencia guía la acción. La investigación previa muestra como la actividad cognitiva y el procesamiento de la información aumentan cuando es necesario restablecer el equilibrio cognitivo (p.e. Hass, Katz, Rizzo, Bailey y Moore, 1992) superando la discrepancia y reduciendo el conflicto (p.e. Jonas, Dile y Bromer, 1997). Pero, no solo aumenta la actividad cognitiva, la información relevante sobre el objeto de la discrepancia se elabora de forma sistemática con intención de afrontar el conflicto interno (Briñol et al., 2004). Por lo tanto, las personas deben dedicar esfuerzo y tiempo a resolver la discrepancia. De esta forma, cuando las personas dispusieron de tiempo para elaborar un plan de acción, resolvieron la discrepancia antes de afrontar el ejercicio. Decidieron la formulación de la meta y el cauce de acción para lograrla, pudieron pasar a la acción e implementar la meta.

Por otro lado, cuando las personas no pudieron resolver las discrepancias antes de realizar el ejercicio mermaron su rendimiento significativamente. Tentativamente, pudo deberse al esfuerzo cognitivo dedicado a resolver la discrepancia mientras debían estar resolviendo el ejercicio. También a la tensión provocada por la falta de ajuste entre la meta y el escaso margen de decisión del que disponía el individuo. En todo caso, les resultó complicado comprometerse con la meta. Nunca llegaron a pasar el Rubicón.

Aunque se han obtenido resultados relevantes aún queda mucho trabajo por hacer. Esta línea de investigación invita a realizar nuevos estudios con el fin de esclarecer las relaciones entre las diferentes fases de acción o las estrategias que utilizan los individuos para lograr la meta. Al menos, hay tres estrategias que resultan plausibles: diseñar un plan de acción acorde a la meta, redefinir la meta (p.e., hacer con- 
gruente la meta con un plan de acción disponible) o definir la meta y el plan. La hipótesis de la tensión sugiere que, en futuros trabajos, se deberán incorporar medidas de satisfacción y bienestar, junto a las medidas de rendimiento. De hecho, la tensión hacia la consecución de la meta determina estados de satisfacción toda vez que la meta se logre o insatisfacción en caso contrario (Kruglanski, 1996). Además, sería conveniente utilizar modelos estadísticos multinivel, toda vez que estos modelos permiten analizar datos cuando los casos (participantes) están agrupados en unidades de información más amplias (grupos) y se ha tomado medida de ambos (Pardo, Ruiz y San Martín, 2007).

La presente investigación tiene una aplicación potencial en el campo de la psicología del trabajo y de las organizaciones. Tener las metas claras y disponer de los recursos adecuados son dos condiciones que se postulan necesarias y suficientes para lograr objetivos. Incluso, para ciertas teorías como la dirección por objetivos, esta idea constituye su fuente de inspiración (Kaplan y Norton, 2000, 2004; Odiorne, 1972). No obstante, los resultados de estos estudios añaden la necesidad de garantizar que los recursos llegan a las personas en forma de planes de acción objetivamente disponibles y cognitivamente controlables. Ya se ha comentado la dificultad para lograr objetivos cuando una persona entra en la fase de ejecución en una situación disonante.

Desde una perspectiva aplicada, los resultan de estos estudios verifican la importancia de formular las metas de forma clara, más aún cuando se trata de metas organizacionales, es decir, metas no formuladas necesariamente por las personas que las tienen que lograr.
También, recalcan la necesidad de congruencia entre la formulación de la meta y la capacidad para conseguirlas. Pero además, sugieren que las metas pueden lograrse aunque no se disponga de una formulación clara o no se dispongan de competencias aprendidas formalmente. Es más, los resultados advierten, por un lado, que resulta más eficaz disponer de un plan que responder a una meta claramente formulada; por otro, que una meta formulada con ambigüedad puede resultar tan eficaz como una meta clara siempre y cuando se disponga de la posibilidad de formular y diseñar un plan de acción. Las consecuencias de estos resultados en el devenir cotidiano de los grupos de trabajo pueden manifestarse en un escenario bifronte. Por un lado, si las personas no reciben sus metas con claridad, pueden reformularlas, planificar su logro y conseguirlas. Ahora bien, con el riesgo de alcanzar metas no deseables o desconectadas de los objetivos estratégicos. Por otro lado, las organizaciones no deben obviar las aportaciones que personas y grupos de trabajo pueden realizar en el desarrollo organizacional en situaciones de incertidumbre.

\section{Conclusiones}

En definitiva, la investigación sugiere que una instrucción de meta clara conduce al logro de la meta sólo cuando la persona tiene accesible un curso de acción adecuado. Si este no es el caso, la instrucción de meta ambigua resulta más efectiva ya que permite redefinir la meta y diseñar un plan de acción apropiado. Por otro lado, el diseño de un plan de acción conduce al éxito con independencia de la instrucción de meta.

\section{Extended Summary}

Most of the goal theories have commonly established that having clear goals is a required condition in order to achieve objectives. Moreover, goal achievement has been studied by several disciplines. In these series of experiments we will consider the psychologi$\mathrm{cal}$ and organizational approaches relevant for research. The psychological approach, which has been influenced by the contribution of Lewin, Dembo, Festinger y Sears (1944) establishes two main components on the goal achievement process: goal setting and goal striving. In this study we will consider the "goal setting theory" (Latham y Locke, 2007; Locke y Latham, 2002) and the phases of action model (Heckhausen y Gollwitzer, 1987; Gollwitzer, 1990, 1996). In regard of the organizational approach, goals are considered as a key element of organization's strategy; moreover, there is a lot methodology related to group or team work; such as: the cycle of PDCA (Deming, 1982; Shewhart, 1931) and the RADAR logic (European Foundation for Quality in Management, 2009).

The following studies aim to contrast whether or not, if a clear goal instruction is a necessary condition to achieving established goals. Consequently, the following hypotheses were proposed:

Hypothesis 1: Having a clear goal instruction, in contrast with ambiguous instruction, will lead to better performance only if the individuals posses adequate competence to achieve goals.

Hypothesis 2: A clear goal instruction will lead to better performance only if the individuals' posses and action plan; even though they do not have formal training or skills to develop task. 
Hypothesis 3: An ambiguous instruction of the goal is positively related with the achievement of the goal, whenever the individuals are allowed to re-formulated goal and establish an action plan.

\section{General Method}

In order to assess which instruction was interpreted as clear or ambiguous a pilot study was conducted. In this study 27 participants were asked to rate the instructions on a scale from 1 (clear) to 5 (ambiguous). Results from the pilot study revealed that clear instruction "The group has to fill out correctly as many chips as possible" was identified as a clear goal $(M=1.33$; $S D=.62$ ). On the other hand, the instruction "Fill out correctly as many chips as possible" was considered significantly more ambiguous $(M=3.52 ; S D=1.22)$, $t(26)=-7.35, p<.001$. Moreover, after the pilot study, 3 experiments were conducted in order to contrast hypothesis previously proposed.

\section{Variable and Measurements}

Clarity of goal instruction (studies 1, 2 and 3). Two categories of variable were manipulated: Clear goal instruction (The group has to fill out correctly as many chips as possible) and ambiguous goal instruction (Fill out correctly as many chips as possible).

Training on team work (analysis of studies 1 and 2). Two groups were differentiated; individuals who have participated on prior training on team work and the individuals who lack training.

The variable planning (study 3). Two alternatives were provided; time was allowed to coordinate actions in achieving goal, and time was not provided.

Goal achievement, based on the total of errors and the mean of this variable.

Controlled variables: The experience with the game was defined by four categories: without experience, with experience at the beginner level, with experience on a medium level, and experience with difficult Sudokus. The need of cognition was measured with the Spanish adaptation (Falces, Brinol, Sierra, Becerra \&Alier, 2001) of the Need for Cognition scale by Cacioppo and Petty (1982). To rule out the fact that participants with higher level of social skills could obtain better results, the Rathus Assertiveness Scale (1973) was also used.

\section{STUDY 1}

In this study we tested if clear goal instruction will trigger better performance, in comparison with the ambiguous instruction. Sixteen volunteer professionals from different business ( 8 females and 8 males) who were getting training on team work participated. The mean age of the participants was 25 years $(S D=.93)$. The present study used a one-factor design with between -factor instruction conditions (clear goal, ambiguous goal). The group error (total number of errors in the board) was used to assess goal achievement. According to the test Mann- Whitney, there were significant differences regarding achievement $(U=.000 ; p<.005)$. Moreover, by the analysis of covariance, the effect of variables of need of cognition and social skills were not relevant. On the other hand, the variable training has direct effects on the dependent variable, consequently they need to be controlled $[F$ $(1,11)=10.900 ; p=.007]$. The ANCOVA test, after eliminating the effect of co-variables, showed effect of the type of the instruction of the goal on the overall achievement of the group $[F(1,14)=303.643 ; p<.001]$. Clear instruction of the goal was preferred (see table 2).

\section{STUDY 2}

In this study, we wanted to address again, whether a clear goal instruction improves goal achievement in contrast with an ambiguous goal instruction However, in this study the participants did not have any prior experience on team work. Eighty volunteer students from the Universidad Autónoma de Madrid participated in research. Fourteen of the participants were males and sixty six females from 18-49 years old $(M=21.85$, $S D=4.44)$. The design and the procedure were the same as in the study 1 . However, in this study participants without prior experience or training on team work were selected. After completion of experiment, an analysis of study 1 and 2 was performed by using factorial analysis inter-subject design 2 (goal instruction condition: clear or ambiguous) $x 2$ (training on team work conditions: with experience and without experience on team work). The t-test for independent samples, demonstrated significant effect of the condition goal instruction (see Table 2). In fact, the participants assigned to the condition of clear goal instruction had more errors $[t(78)=2.81, p=.006]$. The analysis of covariance contrasted the effects of the instruction of the goal and the achievement of it $[F(1,75)=$ $\left.51.533 ; p=.011 ; \eta^{2}=0.083\right]$. No effects were found on the controlled variables. Moreover the parallel analysis of experiments 1 and 2 used the analysis of covariance $2 \times 2$ which revealed a significant effect of the instruction of the goal $\left[F(1,89)=5,75 ; p=.019 ; \eta^{2}=.061\right]$. For the variable prior training in team work, significant differences among groups were evident $[F(1,89)=$ $\left.7.984 ; p=.006 ; \eta^{2}=.082\right]$. And the significance of the interaction was also evidenced. $[F(1,89)=23.240$; $\left.p<.001 ; \eta^{2}=.207\right]$. The lack of prior training on team work has found: a) the relationships between clear goal 
instruction and higher levels of achievement needs to be discarded; b) ambiguous goal instruction has trigger better results; and c) having or not having prior training of team work is not a relevant variable for individuals whenever they receive ambiguous instruction.

\section{STUDY 3}

On this experiment, hypothesis 2 and 3 are contrasted. Sixty four volunteer graduate students from Universidad Autónoma de Madrid participated on study, 50 females and 14 males between the ages of 18 and $32(M=22.06, S D=3.14)$. None of the participants, had prior experience on team work. This study uses a $2 \times 2$ factorial design with the between factors goal instruction condition (clear goal instruction, ambiguous goal instruction) and planning condition (planning, no planning). The analysis of covariance revealed the main effect of the clarity goal instruction; $\left[F(1,57)=16.896 ; p<0,001 ; \eta^{2}=.229\right]$. The main effect of the variable planning was also significant $[F$ $\left.(1,57)=34.847 ; p<0,001 ; \eta^{2}=.379\right]$, (see table 2). Moreover, the results revealed an effect of the interaction of both variables previously described $[F(1,57)=$ $\left.31.209 ; p=.03 ; \eta^{2}=.080\right]$, (see fig. 3). By contrasting both conditions of the variable clarity of goal instruction, we observed that the individuals who had the opportunity to plan were more successful. Once again it has been observed the fact that clarity in goal instruction does not guarantee the achievement of it. Moreover, by adding the variable "planning" we have new conclusions: the inability to plan negates the positive effect of clear goal instructions.

\section{General Discussion}

The results presented on this report confirm the validation of hypothesis 1 and 3. We consider that the results of this research are relevant due to 3 main reasons: a) they verify that clarity of goal instruction is not always related to the goal achievement; b) we have broaden our knowledge regarding setting of goals, since ambiguous goal instruction is more successful that clear goal instruction on some circumstances; c) these studies provides us with better understanding of the relationship between goal setting and goal striving. These results could help us understand psychological processes such as motivation and will. People's beha-vior tends to find a balance between internal coherence and equilibrium with environment. Clear goal setting is successful whenever the individual has strategies to achieve it. The need of coherence is hidden, and people could go straight to action without the elaboration of a plan. However, it seems that the need of coherence and equilibrium lead our actions. Prior investigations demonstrated how the cognitive activity and information pro- cessing increases whenever there is the need to regain cognitive balance (e.g.,Hass, Katz, Rizzo, Bailey y Moore, 1992) overcoming discrepancies and reducing conflict (e.g. Jonas, Dile, Bronner, 1997). On the other hand, a person spends a great deal of time and effort trying to resolve discrepancies. Consequently, whenever an individual spends time on making an action plan, he or she resolves the discrepancy beforehand.

In conclusion, this research suggests that clear goal instruction will lead to better goal achievement, only if the individual has an adequate action plan. Without an action plan, the ambiguous goal instruction will be more effective; since it allows room for adjustment of the goal and the design of an action plan. Finally, the design of an action plan will lead to better performance independently of the condition of the instruction

\section{Referencias}

Abelson, R.P., Aronson, E., McGuire, W. J., Newcomb, Th.M., Rosenberg, M.J. y Tannenbaum, P.H. (1968). Theories of cognitive consistency: A sourcebook. Chicago: Rand McNally.

Bargh, J. A. y Gollwitzer, P. M. (1994). Environmental control of goal-directed action. En W. Spaulding (Ed.), Nebraska Symposium on Motivation: Integrative views of motivation, cognition, and emotion (Vol. 41, pp. 71-124). Lincoln: University of Nebraska Press.

Beckmann, J., \& Gollwitzer, P. M. (1987). Deliberative versus implemental states of mind: The issue of impartiality in pre- and postdecisional information processing. Social Cognition, 5, 259-279.

Briñol, P., Horcajo, J., De la Corte, L., Valle, C., Gallardo, I., y Díaz, D. (2004). El efecto de la ambivalencia evaluativa sobre el cambio de actitudes. Psicothema, 16, 373-377.

Costa, M. y López, E. (2006) Manual para la ayuda psicológica. Madrid: Pirámide.

De Miguel, J.M.; Gallardo, I.; Horcajo, J; Becerra, A.; Aguilar, P. y Briñol. P. (2009). El Efecto del Estrés sobre el Procesamiento de Mensajes Persuasivos. Revista de Psicología Social, 24, 399-409.

Deming, W. E. (1982). Out of the crisis. Cambridge: Massachusetts Institute of. Technology.

European Fundation for Quality in Management (2009). EFQM Excellence Model 2010 version. Bruselas: European Fundation for Quality in Management.

Falces, C., Briñol, P., Sierra, B., Becerra, A., y Alier, E. (2001). Validación de la escala de necesidad de cognición y su aplicación al estudio del cambio de actitudes. Psicothema, 13, 622-628.

Festinger, L. (1957). A theory of cognitive dissonance. Stanford University Press.

Gollwitzer, P. M. (1990). Action phases and mind-sets. En E. T. Higgins \& R. M. Sorrentino (Eds.), The handbook of motivation and cognition: Foundations of social behavior (Vol. 2, pp. 53-92). New York: Guilford Press.

Gollwitzer, P. M. (1996). The volitional benefits of planning. 
En P. M. Gollwitzer y J. A. Bargh (Eds.), The psychology of action. Linking cognition and motivation to behavior (pp. 287-312). New York: The Guilford Press.

Gollwitzer, P. M., Heckhausen, H., y Ratajczak, H. (1989). Perceived proximity to making a decision. En F. Halisch y J. H. L. van den Bercken (Eds.), International perspectives on achievement motivation (pp. 291-308). Lisse: Swets \& Zeitlinger.

Hass, R.G., Katz, I., Rizzo, N., Bailey, J. y Moore, L. (1992). When racial ambivalence evokes negative affect, using a disguised measure of mood. Personality and Social Psychology Bulletin, 18, 786-797.

Heckhausen, H., y Gollwitzer, P. M. (1986). Information processing before and after the formation of an intent. En F. Klix y H. Hagendorf (Eds.), In memoriam Hermann Ebbinghaus: Symposium on the structure and function of human memory (pp. 1071-1082). Amsterdam: Elsevier/ North Holland.

Heckhausen, H., \& Gollwitzer, P. M. (1987). Thought contents and cognitive functioning in motivational versus volitional states of mind. Motivation and Emotion, 11, 101120.

Jonas, K., Diehl, M. y Bromer, P. (1997). Effects of attitudinal ambivalence on information processing and attitude-intention consistency. Journal of Experimental Social Psychology, 33, 190-210.

Kahneman, D. (2003). Maps of Bounded Rationality: Psychology for Behavioral Economics. American Economic Review, 93, 1449-1475.

Kaplan, R. S. y Norton, D. (2000). Cuadro de mando integral: the balanced scorecard. Barcelona: Gestión 2000.

Kaplan, R. S. y Norton, D. (2004). Mapas estratégicos. Barcelona: Gestión 2000.

Karasek, R. (1979). Job demands, job decision latitude and mental strain: Implications for job redesign. Administrative Science Quarterly, 24, 285-306.

Kjormo, O. y Halvari, H. (2002). Two ways related to performance in elite sport: The path of self-confidence and competitive anxiety and the path of group cohesion and group goal-clarity. Perceptual and Motor Skills, 94, 950-966.

Kruglanski, A. W. (1989): Lay epistemics and human knowledge. Cognitive and motivational bases. Plenum Press, New York.

Kruglanski, A. W. (1996). Goals as knowledge structures. In P. M. Gollwitzer \& J. A. Bargh (Eds.), The psychology of action: Linking cognition and motivation to behavior (pp. 599-618). New York: Guilford.

Kruglanski, A. W. y Klark, Y. (1985): "Knowing what to do: On epistemology of actions". En J. Kuhl y J. Beckmann (Eds.), Action control. From cognition to behavior (pp. 4160). Springer, Berlín.

Latham, G. P. y Locke, E. A. (2007). New developments in and directions for goal-setting research. European Psychologist, 12, 290-300.

León, O. y Montero, I. (2001). Cómo explicar el concepto de interacción sin estadística: análisis gráfico de todos los casos posibles en un diseño $2 \times 2$. Psicothema, 13, 159165.

Lewin, K., Dembo, T., Festinger, L. y Sears, P.S. (1944). Level of aspiration. En J. McV. Hunt (ed.): Personality and the Behavior Disorders (pp. 333-378). Nueva York: Ronald Press.

Locke, E. A. y Latham, G. P. (2002). Building a practically useful theory of goal setting and task motivation. A 35-year odyssey. American Psychologist, 57, 705-717.

Locke, E. A., Shaw, K. N., Saari, L. M. y Latham, G. P. (1981). Goal setting and task performance: 1969-1980. Psychological Bulletin, 90, 125- 152.

McGrath, J.E. (1976). Stress and behaviour in organizations. En M.D. Dunnette (Ed.), Handbook of industrial and organizational psychology. Chicago: Rand McNally.

Nemanich, L. A. y Keller, R. T. (2007). Transformational leadership in an acquisition: A field study of employees. Leadership Quarterly, 18, 49-68.

Odiorne, G. S. (1972). La dirección por objetivos. Barcelona: Labor.

Oettingen, G. y Gollwitzer, P. M. (2001). Goal setting and goal striving. En A. Tesser y N. Schwarz (Eds.), The Blackwell handbook of social psychology (pp. 329-347). Oxford: Blackwell.

Oettingen, G., Grant, H., Smith, P. K., Skinner, M. y Gollwitzer, P. M. (2007). Nonconscious goal pursuit: Acting in an explanatory vacuum. Journal of Experimental Social Psychology, 42, 668-675.

Pardo, A.; Garrido, J; Ruiz, M.A. y San Martín, R. (2007). La interacción entre factores en el análisis de varianza: errores de interpretación. Psicothema, 19, 343-349.

Pardo, A., Ruiz, M. A. y San Martín, R. (2007). Cómo ajustar e interpretar modelos multinivel con SPSS. Psicothema, 19, 308-321.

Peiró, J. M. y Salvador, A. (1993). Control del estrés laboral. Madrid: Eudema..

Quinn, J.B. (1993). El concepto de estrategia. En H. Minztberg y J.B. Quinn. El proceso estratégico. Conceptos, contextos y casos. México: Prentice Hall.

Rathus, S.A. (1973). Escala de asertividad. En P. Bartolomé, J.A. Carboles, M. Costa, T. del Ser (Eds.). (1979). La práctica de la terapia de conducta. Madrid: Pablo del Río (apéndice).

Suzuki E., Kanoya Y., Katsuki T. y Sato C. (2007). Verification of reliability and validity of a Japanese version of the Rathus Assertiveness Schedule. Journal of Nursing Management, 15, 530-537.

Shewhart, W. A. (1931) Economic Control of Quality of Manufactured Product. Princeton: Van Nostrand Reinhold Co.

Taylor, S. E. (1982). Social cognition and health. Personality and Social Psychology Bulletin, 8, 549-562.

Tversky, A. y Kahneman, D. (1974). Judgment under uncertainty: heuristics and biases. Science, 185, 1124-1131.

Manuscrito Recibido: 15/09/2009

Revisión Recibida: 05/11/2009

Aceptado: 15/11/2009 